\title{
Entrevistas Clínicas en la Investigación Educativa: un análisis desde la progresividad discursiva
}

\author{
Clinical Interviews in Educational Research: \\ an analysis based on discourse progressiveness
}

Enrique Andrés Coleoni ${ }^{1}$

Laura María Buteler ${ }^{1}$

'Universidad Nacional de Córdoba, Instituto de Física Enrique Gaviola, Consejo Nacional de Investigaciones
Científicas y Técnicas, Córdoba, Argentina.
Autor Correspondiente: enrique.coleoni@unc.edu.ar

Resumen: Se indaga sobre las improvisaciones que un entrevistador lleva a cabo durante la entrevista clínica orientada a obtener datos sobre el aprendizaje en Física. Se utilizaron registros de dos entrevistas que permitieron obtener datos de distinta calidad. Se caracterizan los tipos de intervenciones del entrevistador según su función: Socialización, Empatía, Introducción y Focalización. El análisis más potente se logra al incorporar la idea de discurso progresivo y un modelo para el mismo desarrollado por Marc Clarà. El estudio arroja resultados en dos niveles. Desde lo metodológico, avanza en entender cómo algunas maneras de intervenir posibilitan entrevistas más o menos productivas. En cuanto a la interacción discursiva, se arroja luz sobre aspectos de la entrevista, tomando como insumo un constructo teórico del discurso en las aulas. Dadas algunas similitudes en las formas de interacción, algunos de estos resultados podrían eventualmente constituir un aporte para analizar el aprendizaje en el aula.

Palabras clave: Enseñanza de la física; Entrevista clínica; Progresividad discursiva; Resolución de problemas.

Abstract: This paper probes into the ways interviewers need to improvise as they carry out clinical interviews that pursue data on the learning of Physics. The records used had been obtained previously and allowed to produce data of different quality for the study. Interventions are typified according to their function (Socialization, Empathy, Introduction and Focalizing). A more compelling analysis is achieved by incorporating the idea of discourse progressiveness and a model for carrying it out that has been developed by Marc Clarà. The study delivers results at two different levels. As for methodology, understanding is advanced on how certain modes of intervention allow interviewers to obtain more or less valuable data. As for discursive interactions, light is shed on some of their characteristics during interviews by taking input from theory developed to describe classroom discourse. Given the interactional similarities between both settings, our results could perhaps feedback into the study of classroom learning.

Keywords: Physics teaching; Clinical interviews; Discourse progressiveness; Problem solving.

Recebido em: 14/04/2020

Aprovado em: 21/08/2020 


\section{Introducción}

Jean Piaget, quien sin duda puede considerarse el precursor de la entrevista clínica, la desarrolló como un método para documentar una conversación abierta mediante la cual podía explorar las maneras en que los niños piensan o explican ciertos fenómenos. Él dedica algunos capítulos de su obra para desarrollar las particularidades de este método (PIAGET, 1926, 1929). Si bien las entrevistas clínicas son un instrumento pertinente para la investigación cualitativa en un amplísimo espectro de áreas de conocimiento, en este trabajo nos interesa la utilidad que este instrumento ha tenido, y tiene, para la investigación educativa en ciencias (BROWN et al., 2018; CARRAHER; SCHLIEMANN, 2002; ELLIS, 2007; GOLDSTONE; WILENSKY, 2008; IZSÁK, 2005; LEMMER; KRIEK; ERASMUS, 2018; TABER; GARCIA-FRANCO, 2010; VOLFSON; ESHACH; BEN-ABU, 2020). Esta herramienta metodológica ha sido extensamente aplicada en la investigación educativa para revelar las ideas que los estudiantes ponen en juego al abordar situaciones que involucran fenómenos y/o conceptos científicos, particularmente en estudios sobre el aprendizaje.

Las tareas y preguntas que el entrevistador prepara con anticipación son el punto de partida de una exploración que busca hacer aparecer detalles clave de los razonamientos de los estudiantes. En el caso del que se ocupa este artículo, estos se vinculan con el estudio del aprendizaje conceptual en física durante la resolución de problemas. Durante la entrevista, otras preguntas necesariamente se deben improvisar: la variedad de ideas que los sujetos ponen en juego es suficientemente rica y amplia como para que puedan ser conjeturadas completamente de antemano. Cada sujeto, o grupo de sujetos entrevistados, produce razonamientos sutil o sustancialmente diferentes entre sí a partir de un mismo problema. La improvisación por parte del entrevistador no sólo resulta inevitable, sino que puede incluso determinar que en una entrevista se obtengan (o no) datos valiosos para la investigación. Este estudio se realiza sobre registros de dos entrevistas llevadas a cabo para estudiar el aprendizaje durante la resolución de problemas. Por lo tanto, "mejores datos" son aquellos que permiten inferir más resultados sobre ese aprendizaje. Tanto la práctica de entrevistar, como el análisis posterior de los registros van construyendo en un entrevistador una habilidad cada vez más desarrollada, un conjunto de estrategias, implícitas y explícitas, que le permiten llevar adelante entrevistas con mayor probabilidad de obtener buenos datos.

Nuestro objetivo es profundizar en esa habilidad, decodificarla, para hacer un aporte de valor metodológico para investigadores en formación.

\section{Entrevistas Clínicas en la Educación en Ciencias y sus Interpretaciones}

Numerosos autores asumen que durante la entrevista clínica se pone en juego (y se puede investigar) un fenómeno cognitivo atribuido exclusivamente al sujeto entrevistado (HAMILTON, 1996; LAMBERG; MIDDLETON, 2009; NAVARRO, 2014; PAPAGEORGIOU; JOHNSON, 2005). El foco de la atención está puesto en las respuestas de los entrevistados. Se asume una relación bastante directa entre lo que el entrevistado sabe y lo que dice. Esta visión de la entrevista clínica obedece a un enfoque individual 
de la cognición (BROWN et al., 2016). Su propósito es develar las creencias, ideas, concepciones de cada individuo. Esta relación entre lo que se 'sabe' y lo que se 'dice' no se toma totalmente a la ligera, y el análisis de los datos a veces incluye una consideración de algunos presupuestos (POSNER; GERTZOG, 1982), o se discuten los procesos inferenciales, por ejemplo, en los protocolos de pensamiento en voz alta de Ericson y Simon (1983). Más allá de que en ocasiones se consideran los procesos que median entre las ideas y las verbalizaciones de los sujetos, se asume que estas reflejan sus representaciones sobre el fenómeno en cuestión (CHI, 1997).

Sin embargo, hoy también se concibe a la entrevista como un fenómeno social además de individual (BUTELER; COLEONI, 2014; COLEONI; BUTELER; BAUDINO, 2016; DiSESSA, 2007; HALLDÉN; HAGLUND; STROMDAHL, 2007; MORTON, 2012; ODDEN; RUSS, 2005; ROTH, 2008; RUSS; LEE; SHERIN, 2012; RUSS; SHERIN; LEE, 2016). Este fenómeno social incluye, por ejemplo, las distintas formas en que entrevistados y/o entrevistador están interpretando la actividad que Ilevan a cabo. En el lenguaje de Goffman (1974), esas formas de entender socialmente la situación son los enmarques que ponen en juego los sujetos involucrados en una situación dada y que responden (implícitamente) a la pregunta: ¿Qué se supone que debo hacer en esta situación? Estos enmarques conllevan un conjunto de metamensajes que actúan y condicionan las respuestas de los entrevistados y las preguntas del entrevistador. Roth (2008) utiliza las entrevistas clínicas para estudiar las ideas intuitivas de los estudiantes mediante una propuesta que desafía el abordaje de la cognición individual y presupone que el medio en el que surgen esas ideas es lo que se habla (talk, en su idioma original). Lo estudiado es el lenguaje que se produce y se reproduce en esa actividad social. Halldén, Haglund y Stromdahl (2007) proponen que la entrevista se puede concebir como un escenario en el cual el entrevistado pone en juego recursos orientados-a-la-tarea (competence-oriented, en el original) y también recursos orientados-al-discurso (discourse-oriented). Estos recursos responden, respectivamente, a lo que el sujeto sabe en relación a qué hacer para resolver una dada tarea y a lo que estima más adecuado en el contexto particular en que resuelve. Buteler y Coleoni (2014) estudiaron los abordajes comunicativos (MORTIMER; SCOTT, 2003) establecidos entre el entrevistador y un grupo de estudiantes universitarios entrevistados mientras resolvían un problema de hidrostática. Paralelamente analizaron, desde un abordaje cognitivo individual (DiSESSA; SHERIN, 1998), cómo estos estudiantes realizaban progresos conceptuales en relación al concepto de empuje. En las conclusiones señalan que los patrones de abordaje comunicativo desarrollados por Mortimer y Scott (2003) para entender el discurso en el aula de ciencias se ajustaron bien al abordaje comunicativo establecido en la situación de entrevista grupal, revelando así su carácter socio-comunicativo.

Similarmente, Russ, Lee y Sherin (2012), analizaron cómo un conjunto de estudiantes secundarios enmarcaba la situación de entrevista mientras respondían individualmente preguntas sobre química básica e identificaron tres patrones distintos: indagación, examen oral y entrevista experta. Los patrones podían cambiar durante la entrevista luego de ciertas intervenciones por parte del entrevistador. Odden y Russ (2019) profundizan sobre cómo ciertas preguntas 'desconcertantes' (vexing questions, en el original) juegan un papel en disparar y mantener patrones de tipo indagación en los estudiantes entrevistados. En el mismo sentido, Shaban y Wilkerson (2019) estudian los cambios en estos tipos de enmarque que espontáneamente pueden ocurrir durante 
las entrevistas. Estos trabajos evidencian una cuestión crítica: la importancia de atender cuidadosamente a la manera en que los estudiantes enmarcan la situación de entrevista.

Russ, Sherin y Lee (2016) analizan un conjunto de entrevistas para recabar ideas intuitivas de niños sobre las estaciones del año. Allí distinguen dos desafíos que el entrevistador debe enfrentar permanentemente mientras conduce la entrevista: el desafío del conocimiento y el desafío de la interacción. El primero se refiere a la necesidad de preservar la ecología conceptual de los entrevistados, sin que sea alterada por las sugerencias o intervenciones del entrevistador (DiSESSA, 2007). El segundo, el de la interacción, se refiere a que los entrevistadores deben evitar que los entrevistados se sientan evaluados (examinación oral en términos de Russ, Lee y Sherin (2012) apegándose a una versión escolar y autoritativa de la situación de entrevista.

Los antecedentes mencionados muestran que el análisis de una entrevista involucra necesariamente una mirada socio-cultural. En general, el foco de estos análisis socio-culturales ha estado puesto en las producciones, posicionamientos, dificultades y avances de los estudiantes entrevistados. En este trabajo, esa mirada socio-cultural incorporará y enfocará sobre el entrevistador como objeto de análisis, con el fin de caracterizar esa habilidad que pone en juego cuando improvisa y navega ese escenario que se conforma a partir de una situación inicial y de sucesivas acciones de los estudiantes entrevistados. El análisis se sostendrá en registros obtenidos durante dos entrevistas, contrastantes entre sí. En ese marco, las preguntas que guían esta investigación son:

- ¿Qué tipos de intervenciones realiza el entrevistador durante cada una de estas entrevistas, y cómo éstas se distribuyen en el tiempo?

- ¿Cómo se articulan esos tipos de intervenciones con el discurso producido durante la entrevista?

\section{Metodología}

El corpus de datos son los registros audiovisuales de dos entrevistas llevadas a cabo en el marco de otra investigación para entender cómo los estudiantes aprenden durante la resolución de problemas de hidrostática. Estas entrevistas fueron elegidas por haber permitido obtener datos bien diferenciados en cantidad y calidad. En la entrevista 1 se obtienen registros más ricos que en la entrevista 2 en términos del aprendizaje que desarrollan los sujetos al resolver los problemas.

Los entrevistados eran estudiantes de una Licenciatura en Física en una universidad pública en Argentina de dos cohortes diferentes cursando el mismo curso de Física Introductoria en el cual se desarrollan contenidos sobre hidrostática. Ambos grupos de estudiantes tenían trayectorias de formación y desempeño académico comparables. En ambos casos, ya habían sido evaluados en los contenidos relativos a hidrostática.

El análisis consta de dos estadios consecutivos. En primer lugar, se indaga sobre el tipo de intervenciones que realiza el entrevistador y la distribución temporal de éstas a lo largo de la entrevista. En una primera inspección, se ubicaron temporalmente todas las intervenciones del entrevistador, tomando como criterio amplio el simple hecho de que el entrevistador hablara. Se utilizó el software Videograph (TM), de análisis de video registros. Al llevar a cabo este primer paso se advirtió la existencia de diferentes tipos de intervenciones, según las formas de expresión y los propósitos a los que servían. Estas intervenciones fueron clasificadas según su función y características. En sucesivas 
discusiones entre los autores se propusieron, cuestionaron, reconsideraron, modificaron y finalmente se acordaron cuatro categorías: intervenciones de empatía, de socialización, de introducción, y de focalización. En la sección siguiente se describen estas categorías.

En una segunda etapa, se analiza cómo se articulan esas intervenciones con el discurso producido durante la entrevista. Se recurre a un modelo teórico para la progresividad del discurso (CLARÁ, 2019) que se implementa en este caso en el contexto de entrevista. Este modelo arroja luz a la problemática planteada porque permite develar dos juegos distintos entre las intervenciones del entrevistador y el discurso resultante, que explicaría la diferencia entre ambas entrevistas.

\section{Resultados}

\section{Parte I: sobre los tipos de intervenciones del entrevistador y su distribución temporal}

Las Intervenciones de Empatía (IE) ayudan al entrevistador a sostener un ambiente de interacción en el que los entrevistados perciben que lo que tienen para decir es de interés para él. El término empatía hace referencia a que el entrevistador está entendiendo sus razonamientos. Muchas veces tienen la forma de frases de asentimiento, como ajá, ok, ahhh, entiendo. El tono de voz es apenas el suficiente para manifestar que se entiende.

Las Intervenciones de Socialización (IS) sirven para promover interacciones entre los entrevistados que potencialmente pueden dar lugar intercambios fructíferos. El ejemplo más común es el de pedir a un entrevistado que opine sobre lo que otro está diciendo, o que manifieste verbalmente un posible desacuerdo que se percibe en el plano gestual. En lo expresivo, las IS tienen características similares a las IE. EI tono de voz es suave, y se eleva tan sólo levemente para hacer notar, por ejemplo, que un(a) compañero/a podría no acordar con lo que se dice.

Las Intervenciones de Introducción (II) permiten al entrevistador poner a disposición de los entrevistados algún elemento que estima potencialmente útil para sus razonamientos, y que los estudiantes no están considerando. Si bien por lo general son enunciadas en forma de preguntas, hay una elevación del tono que permite comunicar una disrupción con lo que el entrevistado está diciendo. El tono permite llamar la atención sobre la novedad de eso que se pone a disposición.

Las Intervenciones de Focalización (IF) ayudan al entrevistador a sugerir que se atienda a alguna cuestión en particular, que aunque ya haya sido mencionada y considerada, pudiera haber quedado en un plano secundario. Las IF sirven para focalizar sobre elementos que ya están presentes en los razonamientos de los estudiantes. Comparten características expresivas con las II: el tono de la voz se eleva para acompañar la intención de focalizar sobre alguna cuestión en particular.

Las dos primeras (IE e IS) tienen funciones fuertemente vinculadas con el plano social. Las dos siguientes (II e IF) tienen una función que incluye aspectos relevantes en el plano del conocimiento conceptual involucrado en las verbalizaciones. La Tabla 1 muestra las categorías construidas, las funciones que se le atribuyen y las definiciones que las operativizan. 
Tabla 1 - Resumen de las funciones y características expresivas de las intervenciones identificadas

\begin{tabular}{|c|c|c|}
\hline Denominación & Función & Características expresivas \\
\hline Empatía (IE) & $\begin{array}{l}\text { Manifestar interés del entrevistador por la tarea del } \\
\text { entrevistado. }\end{array}$ & \multirow{2}{*}{$\begin{array}{l}\text { El tono de voz es suave, acompaña la } \\
\text { actitud de aceptar sin cuestionamiento } \\
\text { lo que los entrevistados expresan. }\end{array}$} \\
\hline Socialización (IS) & $\begin{array}{l}\text { Promover interacciones entre los entrevistados (dar } \\
\text { opinión, acordar, disentir). }\end{array}$ & \\
\hline Introducción (II) & $\begin{array}{l}\text { Poner a disposición de los entrevistados un elemento } \\
\text { que no está presente en sus razonamientos. }\end{array}$ & \multirow[t]{2}{*}{$\begin{array}{l}\text { El tono de voz se eleva para acompañar la } \\
\text { novedad de lo que se introduce o focaliza. }\end{array}$} \\
\hline Focalización (IF) & $\begin{array}{l}\text { Llamar la atención sobre algún elemento que, aún } \\
\text { estando presente, no está siendo considerado. }\end{array}$ & \\
\hline
\end{tabular}

Fuente: elaboración de los autores.

Las Figuras 1 y 2 muestran, respectivamente, el mapa temporal de las distintas intervenciones en cada entrevista. En la Figura 1 parece no haber IS, y en la figura 2 parece no haber intervención alguna hasta el minuto 15, aproximadamente. Esto es un efecto de la escala temporal (que permite ver la entrevista completa). No se advierten esas intervenciones porque las líneas que las marcan son demasiado delgadas (intervenciones cortas en duración).

Figura 1 - Distribución de los diferentes tipos de intervenciones en la primera entrevista

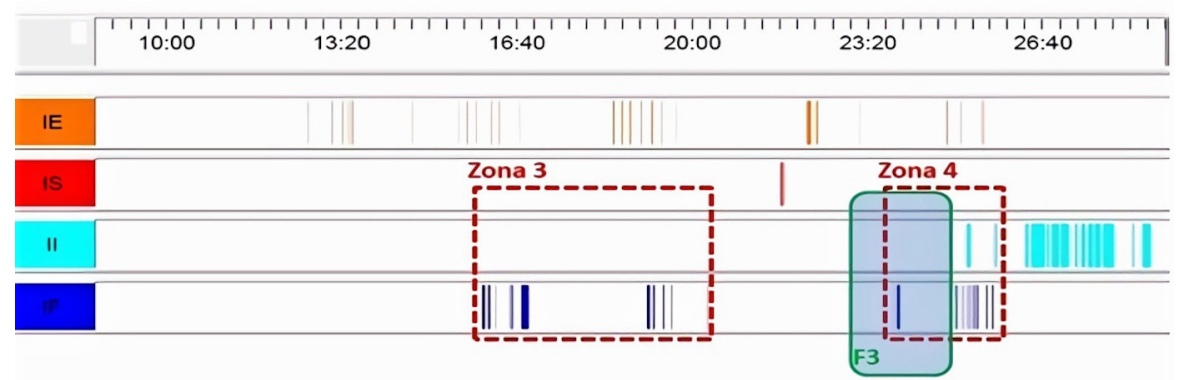

Leyenda: IE: Intervenciones de Empatía; IS: Intervenciones de Socialización; II: Intervenciones de Introducción; IF: Intervenciones de Focalización. Las IS no se visualizan por la escala del gráfico, pero sí ocurrieron, y por eso se incluyen en la codificación. Las Zonas 1 y 2 refieren al análisis de la distribución de intervenciones. F1 y F2 señalan los fragmentos analizados en la Parte II de la esta sección.

Fuente: elaboración de los autores.

Figura 2 - Distribución de los diferentes tipos de intervenciones en la segunda entrevista

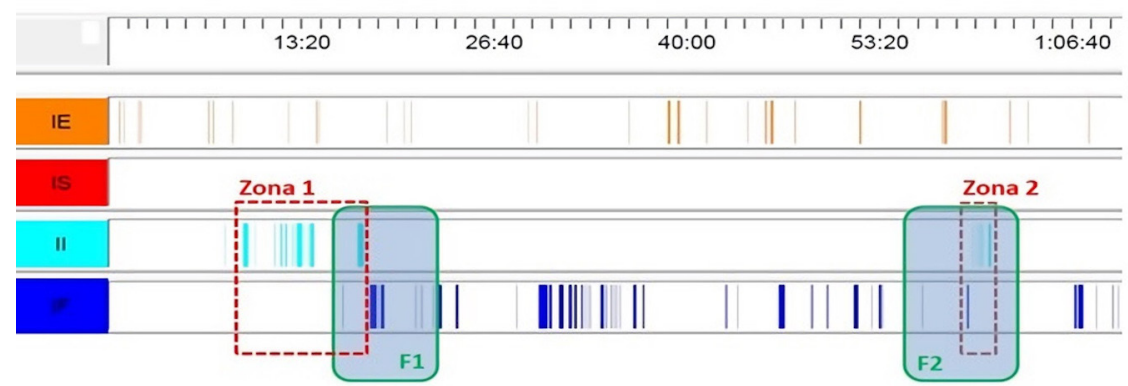

Leyenda: IE: Intervenciones de Empatía; IS: Intervenciones de Socialización; II: Intervenciones de Introducción; IF: Intervenciones de Focalización; En los primeros 15 minutos de entrevista sí ocurrieron intervenciones de empatía que no se visualizan por cuestiones de escala. Las Zonas 3 y 4 refieren al análisis de la distribución de intervenciones. F3 señala el fragmento analizado en la Parte II de la esta sección.

Fuente: elaboración de los autores. 
Las diferencias más significativas entre ambas entrevistas están en la distribución de las II y de las IF. En la entrevista 1 hay un lapso dominado por la presencia de IF, flanqueado por dos zonas en las cuales hay abundancia de II (Zona 1 y Zona 2, figura I). La entrevista 2 presenta dos zonas con abundancia de IF (Zona 3 y Zona 4, figura 2). La Zona 4 se superpone con un lapso de presencia marcada de II. Esto parecería estar indicando otra dinámica de conducción en la entrevista.

Así pues, en términos de distribución, lo que distingue más claramente a las entrevistas es esta manera diferente de acumularse las II y las IF. Decidimos entonces analizar qué función estaban jugando estas intervenciones en ambas entrevistas.

\section{Parte II: la articulación con el discurso}

Para abordar la segunda pregunta de investigación, se utilizó un modelo para la progresividad discursiva (CLARÀ, 2019). El mismo se discutirá primero, antes de pasar a los resultados.

\section{a. Un modelo para la progresividad discursiva}

La idea de discurso progresivo (BEREITER, 1994) surge para superar una interpretación común del posmodernismo en la enseñanza de la ciencia. Si el aprendizaje por indagación ha de respetar el principio de que todo el conocimiento se debe construir exclusivamente a partir de las creencias de los estudiantes, se excluye la posibilidad de que haya un punto de vista objetivo para juzgar si algo es cierto no, y por lo tanto no puede haber ningún progreso posible porque no hay manera de demostrar que cualquier afirmación es más válida que otra. En contraposición a estas ideas, Bereiter (1994) propone que el supuesto clave de la indagación científica no es la verdad objetiva sino la progresividad, y que ésta se juzga en términos de si quienes participan de la indagación están de acuerdo en que las nuevas ideas superan a las viejas. De esta manera, el discurso progresivo permite dar respuesta a cómo el conocimiento científico logra avanzar, mediante la interacción entre pares, antes de contar con una verdad objetiva con la cual contrastar los enunciados que produce.

Recientemente, Clarà (2019) propone un mecanismo para la progresividad en el discurso dialógico. Analiza la progresividad estudiando la manera en que los hablantes desarrollan una estructura semántica común (ESC). Al estudiar el desarrollo de esa ESC, él identifica un mecanismo que consiste en una serie de iteraciones de la secuencia Dirección $\rightarrow$ Inferencia $\leftrightarrow$ Observación $\leftrightarrow$ Consenso $\rightarrow$ Fijación.

Dirección: la dirección es la pregunta misma que genera la discusión. Es aquello que dispara el proceso de diálogo entre las diferentes ideas de los estudiantes. Por ejemplo, en el caso del problema 2, la dirección está marcada por la pregunta sobre si la pelota subirá o bajará al agregar aceite sobre la superficie libre del agua. Es una pregunta que no tiene respuesta en el marco del esquema de significados compartidos hasta ese momento.

Inferencia: proponen una estructura semántica $(E S)^{1}$ que intenta dar respuestas

'La ES y la ESC son básicamente la misma cosa. Una estructura semántica alcanza el estatus de Común sólo cuando ha sido alcanzado un consenso. Hasta entonces, la ES puede estar siendo negociada, como se verá en los resultados. 
a la pregunta dada (la Dirección). Es una afirmación acerca de cómo será o no el comportamiento del sistema físico considerado. Por ejemplo, en el caso del problema 2, a poco de leer el problema, uno de los entrevistados, C, manifiesta "Yo estoy convencida que el aceite le hace fuerza para abajo a la pelota, de alguna forma... le tiene que hacer peso". Esta es una Inferencia, una afirmación que se pone a consideración como respuesta plausible para la pregunta manifestada en la Dirección.

Observación: las Observaciones pueden confirmar, modificar, o rechazar las Inferencias. Pueden consistir en una simple afirmación en oposición a la inferencia, o en algún argumento que plantea su invalidez. En el problema 1, al considerar la relación entre peso y empuje sobre el cubo sumergido en el líquido, los estudiantes han propuesto la inferencia de que bajo el cubo no hay agua. Esta inferencia es observada por el hecho de que "antes no se fijaban si había agua o no, sólo en el volumen desplazado". Esa interacción entre Inferencias y Observaciones es exitosa si entre todos los participantes se alcanza un consenso.

Consenso: es la parte del proceso en la cual los participantes alcanzan un acuerdo sobre las diferentes características de la ES (y por eso, esta se denomina entonces ESC), o acuerdo sobre una estructura semántica, que pasa así a ser común.

Fijación: es un dispositivo que permite materializar el consenso alcanzado como, por ejemplo, una ecuación o un dibujo. Es una ratificación explícita de esa estructura semántica compartida.

Como habíamos señalado anteriormente, el análisis de los tipos de intervenciones y de su distribución temporal no permite dar cuenta cómo un entrevistador logra (o no) obtener datos útiles para su investigación. Ese primer análisis resulta un punto de partida necesario para profundizar la búsqueda mediante el análisis discursivo. Son las II y las IF las que muestran, en ambas entrevistas, una distribución cualitativamente diferente. Estas diferentes distribuciones orientan la mirada a diferentes fragmentos para analizar la progresividad del discurso de los estudiantes, en relación con las intervenciones del entrevistador. A los fines de presentar las características del análisis, y de mostrar los resultados diferenciados obtenidos en ambas entrevistas, se describen los obtenidos en los fragmentos señalados como F1, F2 y F3 en las Figuras 1 y 2.

\section{b. Entrevista 1: intervenciones que favorecen la progresividad}

El primer fragmento analizado corresponde a la entrevista 1, y se ubica en la yuxtaposición entre la Zona 1 (abundante en II) y el lapso subsiguiente, con alta presencia de IF. En la Figura 1 se lo ha señalado como una sombra con el nombre F1 (fragmento 1). A continuación, se transcriben en el cuadro las alocuciones de entrevistados y entrevistador, y a posteriori el análisis correspondiente. Hay una ES inicial, en la cual una inferencia juega un papel central (no hay empuje sobre el cubo de metal en el fondo del recipiente, porque no hay agua debajo de él). A partir de una II se abrirá una Dirección que permitirá observar esa ES a lo largo del fragmento. Mediante dos IF se abrirá un camino que permite a los estudiantes realizar dos Observaciones: a la ausencia de agua bajo el cubo y a la posibilidad de que no haya empuje sobre un cuerpo que está sumergido en un líquido. 
T1 [A] Si no tiene líquido debajo, el empuje no le hace nada.

T2[ENT] ¿Y si fuera una pelotita en vez de un cubo? [II: el problema no invitaba a pensar en una esfera, esta idea fue introducida por el entrevistador]

T3[A] En una pelota, como que en un único punto estaría apoyado el cuerpo sobre la superficie del fondo... y en todo lo otro [gesticula con el dedo la forma curva de la pelota] se levanta sobre el fondo y ahí sí el agua hace una fuerza...

$\mathbf{T 4}[\mathrm{J}]$ ¿Tiene agua por debajo en ese caso?

T5[ENT] ¿Qué quiere decir 'tiene agua por debajo'? a ver si yo entiendo bien. [IE]

T6[J] Claro, porque nosotros habíamos supuesto que ese cubo estaba totalmente en contacto con la superficie [gesticula con la palma extendida sobre la superficie de la mesa] entonces no queda nada de agua entre la superficie inferior del cubo y el recipiente.

T7[ENT] Aja... o sea... ¿está seca la base del cubo? [IF: focaliza sobre lo que implica pensar que no hay agua bajo el cubo]

T8[J] Sí... [se ríe... no está cómodo aceptando que 'no hay agua' equivale a 'está seca la base del cubo']

T9[A] Lo que nosotros decimos es que tenés el recipiente así, y la bolita así [con la mano representa una superficie plana y encima una curvada] y entonces, acá, hay agua.

T10[J] En ese caso [una esferita] sí tendría empuje...

T11[M] Pero nunca nos fijábamos si tenía agua...

T12[J] No, claro, el empuje no depende de la forma, nomás depende del volumen...

T13[ENT] Entonces, en este caso, [señala el cubo] acá ¿sí hay empuje o no hay empuje...? [IF: focaliza sobre una incompatibilidad que ya han notado]

$\mathbf{T 1 4}[\mathbf{M}, \mathbf{J}]$ Está sumergido, así que desplaza algún volumen de agua...

T15[J] Te acordás cuando veíamos el 'porqué' del empuje, era porque el volumen de agua desplazado, era sostenido por el agua que tenía debajo... entonces sí importa que haya agua debajo o no...

T16[M] Pero, a ver... [dibujando] si tenemos un cubito acá, y tenemos una pequeeeeña laminita de agua.

T17[J] Pero el empuje es una fuerza que hace el líquido para sostener ese cuerpo que está ahí porque desplazó por eso depende del volumen de agua desplazado.

T18[ENT] $Y$ en este caso [señala el cubo] ¿no hay volumen de agua desplazado? [IF: los estudiantes ya habían mencionado el volumen de agua desplazado]

T19[J] Sí, pero no está sustentado por nada [de líquido] debajo.

T20[ENT] O sea, la bolita tiene empuje, pero el cubo... [IF: focaliza sobre dos ideas contrapuestas que consideran al mismo tiempo]

T21 [J] No! con lo que acabamos de decir, los dos tienen que tener...

Comenzaremos por determinar en qué medida este fragmento presenta características progresivas. Efectivamente, al comienzo, la ES se sintetiza en la alocución de $A$, en el turno 1: no hay líquido bajo la cara inferior del cubo, y por lo tanto no hay empuje sobre el cubo. Hacia el final del fragmento, el sentido que están negociando los estudiantes navega entre dos estructuras que no coinciden entre sí: por un lado, el empuje es ejercido por el líquido que 'sostiene' al cuerpo; en contraposición, el empuje es una fuerza que depende de la cantidad de fluido desplazado, independientemente de la geometría del mismo. La ES está siendo modificada.

En segundo lugar, analizaremos qué papel juegan las intervenciones del entrevistador. En el turno 2, tenemos una II: los estudiantes no habían hecho consideración alguna en relación a lo que sucedería con una esfera. Esta intervención permite establecer una Dirección que dispara un proceso de diálogo entre diferentes ideas de los estudiantes. En el turno 7, hay una IF. Los estudiantes habían explicitado en varias alocuciones la Inferencia "bajo el cubo no hay agua". La IF del turno 7 resalta una consecuencia no advertida de esa Inferencia: "no hay agua" equivale a afirmar 
que la cara inferior (sumergida) está seca. Así, esta IF promueve una Observación a esa Inferencia. La IF del turno 13, retoma esta misma Observación de los estudiantes. Los mismos estudiantes explicitan dos Inferencias que son incompatibles entre sí: si hay volumen desplazado hay empuje (turnos 14 y 17) y si hay empuje debe haber líquido debajo (turnos 15 y 19). Las IF de los turnos 13, 18 y 20 ayudan a manifestar la incompatibilidad entre esas dos Inferencias que están compitiendo para ocupar un lugar en la ES.

En síntesis: mediante una II, el entrevistador ofrece una dirección que promueve una fuerte interpelación a la ES inicial. Mediante posteriores IF el entrevistador: (1) resalta una consecuencia inadvertida de una Inferencia (turno 7), habilitando a que ellos mismos la Observen (turnos 11 y 12); y, (2) permite a los estudiantes explicitar una Observación que ellos ya habían gestado (turnos 13, 18 y 20).

El segundo fragmento analizado corresponde a la zona sombreada como F2 (fragmento 2) en la Figura 1. Veremos cómo mediante ciertas Observaciones se modifica la ES. Al comienzo del fragmento, la discusión entre los estudiantes se plantea en relación a dos posibles escenarios: por un lado, asumir que bajo el cubo de metal no hay líquido, y por lo tanto, la fuerza de contacto que el recipiente ejerce sobre el cuerpo es igual al peso del cuerpo²; por otro lado, asumir que bajo el cuerpo hay una pequeña laminita de agua y entonces la fuerza que es igual en magnitud al peso del cuerpo es el empuje. La disyuntiva planteada deja afuera la posibilidad de que las fuerzas actuantes sobre el cubo sean tres (su peso, una fuerza normal de contacto con el fondo, y el empuje) y sólo admite dos posibles situaciones, en las cuales sólo dos fuerzas actúan sobre el cubo: el peso y la normal (asumiendo que no hay agua), o el peso y el empuje (asumiendo que sí hay agua). Los tres estudiantes acuerdan en que, al estar el cubo sumergido en el fondo, hay un incremento en la lectura de la balanza que es igual en magnitud al empuje (peso del volumen desplazado). En lo que no acuerdan es en la relación de ese empuje con el peso del cuerpo sumergido.

T22[A, M] O sea, la balanza va a estar marcando [de más] el empuje.

T23[M] Que en este caso no... [volviéndose hacia J] [...] no es peso...

T24[J] [...] que es el peso [...]

[Las alocuciones de T23 y T24 ocurren al mismo tiempo. Tanto M como J se detienen, mirándose el uno a la otra, al darse cuenta de que están en desacuerdo]

T25[ENT] ¿El empuje? ¿y la normal? [IF: ya habían mencionado la 'normal correspondiente al cuerpo' para referirse al incremento en la lectura de la balanza al introducir el cuerpo]

T26[J] Me perdí con todas las normales que... [se ríe], o sea...

T27[ENT] Esa normal, es la que viene de... ¿estar en contacto con la base? [IF: focaliza sobre la necesaria relación entre la normal y el contacto, ambos mencionados previamente]

T28[J] Pero si no está en contacto con la base, iporque hay agua!

T29[ENT] Contacto, pero... contacto, no quiere decir necesariamente ... ausencia de agua.... ¿no? Puede haber contacto y también haber agua. [II: introduce la posibilidad de que simultáneamente haya contacto y presencia de agua]

T30[J] Ah... como huequitos y que puede haber agua... yo siempre me lo imagino liso, digamos, y si hay algo de agua en el medio, no hay contacto...

\footnotetext{
${ }^{2}$ Esto no es estrictamente cierto, puesto que si el contacto entre ambas superficies fuera total, esa fuerza de contacto
} sería mayor al peso del cuerpo, dada la presión de líquido ejercida sobre la superficie superior del cubo. 
T31 [A] Bueno, pero imaginate que vos [toma el lápiz y hace el dibujo de la Figura 3] tenés el cubo así macroscópicamente, y vos tenés el fondo acá.

T32[J] [Señalando en el dibujo] Claro, hay partes en las que está en contacto, claro.

T33[A] O sea, en estos puntos, toca... y acá tenés agüita... [señalando en el dibujo.] Claro, nosotros decíamos 'o el peso es igual al empuje, o el peso es igual a la normal'.

T34[A] Nunca considerábamos las tres...

[T33 y T34 son dos maneras de expresar la misma inferencia: hay normal y hay empuje / hay contacto con el fondo, y también hay agua]

T35[J] Nunca considerábamos que fuera rugoso el cubo...

Primero debemos advertir que hay un cambio en la ES. Al comienzo, dos Inferencias con características e implicancias distintas impiden un Consenso. Una de esas Inferencias es que bajo el cubo sólo hay agua, y por lo tanto la única fuerza (hacia arriba) que actúa sobre el cuerpo es el empuje (turnos 24 y 28). Por otro lado, el turno 23 da cuenta de una Inferencia incompatible con la anterior: el peso del cuerpo no puede ser igual al empuje, porque en ese caso, el cuerpo no se hundiría hasta el fondo del recipiente. Hacia el final, hay una ES en la que acuerdan todos los estudiantes, es decir, se convierte en una $E C^{3}$. En esta ESC hay una inferencia que resulta crucial: en la base del cubo hay contacto con el recipiente y al mismo tiempo contacto con el agua a la presión correspondiente en ese punto (turnos 30, 31, 32, 33, 34 y 35).

Figura 3 - Dispositivo de Fijación mediante el cual A, M y J dejan expresado el Consenso al cual llegan

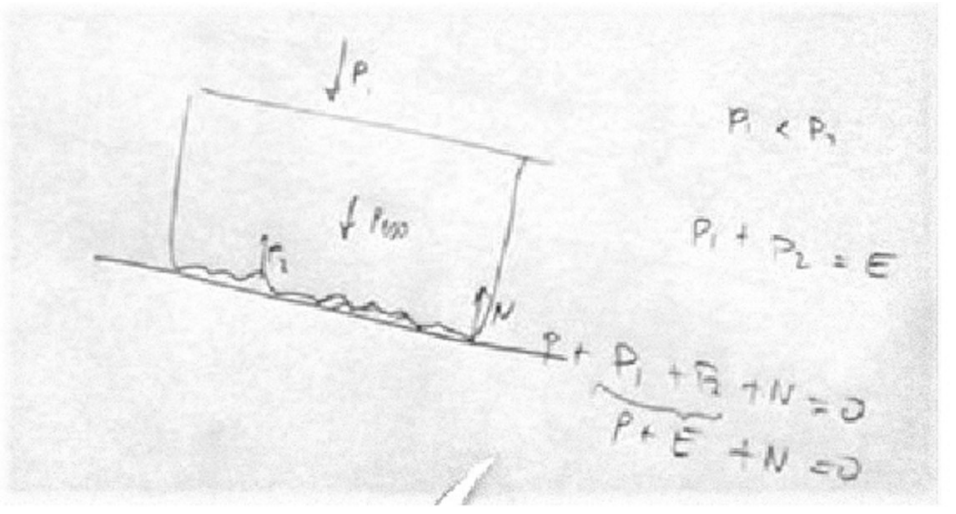

Fuente: elaboración de los autores.

El segundo plano del análisis corresponde a cómo las intervenciones del entrevistador se articulan con esos cambios en la ES. En el turno 25 una IF pone el foco sobre una fuerza que ya había sido mencionada pero que no se está considerando, la Normal. Este movimiento abre la posibilidad de que los estudiantes Observen la Inferencia "bajo el cubo sólo hay agua". Mediante una IF (turno 27), el Entrevistador avanza un paso más en promover esa Observación: contrapone esa Normal (antes mencionada) con la inferencia que "bajo el cubo sólo hay agua". Esta movida logra que J explicite el lugar de esa inferencia en la ES ("Pero si no está en contacto con la base, iporque hay agua!") y así, mediante la II del turno 29 el Entrevistador pone a disposición de $J$ un elemento nuevo que J convierte en Inferencia: puede haber simultáneamente contacto con el fondo y presencia de agua.

${ }^{3}$ Si bien en esta porción no aparece el acuerdo explícito de $M$, esto sí ocurre algo después. 
Estas intervenciones (las IF de los turnos 25 y 27 y la II del turno 29) permitieron abrir un camino de modificación de la ES: Observar la Inferencia "bajo el cubo sólo hay agua" e incorporar una nueva Inferencia, superadora de aquella: "hay, simultáneamente, contacto y presencia de agua". Un indicador interesante de este proceso son las alocuciones de los turnos 34 y 35 . Allí, refiriéndose tanto a la consideración de 3 fuerzas (Peso, Empuje y Normal) en lugar de solo dos, o a la manera de modelar la superficie de contacto, dan cuenta de cómo cambió la ES luego de haber observado una inferencia e incorporado otra. De hecho, estas alocuciones dan cuenta del Consenso que han alcanzado sobre esa, ahora, ESC. Consenso que proceden a fijar en los minutos posteriores mediante dos dispositivos escritos: un dibujo que muestra el contacto entre las superficies a nivel microscópico y la ecuación que refleja la relación entre Peso, Normal y Empuje (Figura 3).

\section{c. Entrevista 2: intervenciones que atentan contra la progresividad}

El análisis se llevó a cabo sobre el fragmento marcado como F3 (Fragmento 3) en la Figura 2. El problema pregunta si una pelota, que flota en agua con $3 / 4$ de su volumen sumergido, subirá o bajará, al echar aceite sobre el agua. Para los estudiantes, la acción de la columna de aceite, ubicada sobre la pelota, es determinante para decidir que la pelota se hundirá más en el agua.

T1 [C] Bueno, entonces [señalando la opción en el enunciado] el objeto se hundirá más en el agua. [N asiente]

T2[C] Por el peso de la columna de aceite.

T3[N] [mientras asiente con la cabeza] Por la presión que recibe la pelota.

T4[C] Claro, [mirando a N] tiene una fuerza más...

T5[N] ... sobre la pelota, sí.

T6[ENT] Una fuerza... ¿hacia abajo? [ellos asienten] [...] Pero el empuje, ¿no es hacia arriba? [IF]

T7[C] Pero no es el empuje esa fuerza, esa fuerza es el peso de la columna de aceite que está sobre la pelota.

T8[ENT] Ah... ok... y entonces... bueno, suponete que ya la cubriste de aceite, y ahí se quedó... y entonces, ahora seguimos agregando... y le echamos, no sé, diez centímetros más de aceite... la pelota, ¿va a cambiar de posición? [IF]

T9[N] [asiente con la cabeza] Aumenta la presión.

T10 [C] [Hace una exclamación de incomodidad y se toma la cabeza con las manos mientras baja la mirada]

[T9 y T10 ocurren al mismo tiempo. $\mathrm{N}$ advierte que $\mathrm{C}$ encuentra una inconsistencia en el razonamiento que él mismo no ve]

T11 [C] [mirando a N] Pero vos decís... si yo le tiro, no sé, un millón de litros de aceite, vos decís que la pelota, va a seguir bajando? [N asiente] porque ya está ahí, hundida... y mientras más aumente yo el peso de la columna de aceite, tendría que ir bajando,... pero no lo veo...

En primer lugar, resulta central para nuestro análisis señalar que al principio del fragmento hay progresividad en el discurso de los estudiantes. Una Inferencia caracteriza la ES: La columna de aceite (sobre la pelota) ejerce una fuerza, independiente del empuje, y la pelota se hunde más. (Turnos 2, 3, 4, 5, 7). En los turnos 10 y 11 , C Observa esa estructura: Si se agrega aceite, la pelota debería hundirse más; sin embargo, si la pelota está completamente sumergida, cambiar la cantidad de aceite no debería cambiar su posición ("no lo veo"). 
Dos IF (turnos 6 y 8) colaboran con esa Observación. En el turno 6, la IF focaliza sobre la dirección del empuje en condiciones de equilibrio hidrostático. La IF del turno 8 focaliza sobre algo que ya habían mencionado (antes de este fragmento transcripto): Aumentar la cantidad de aceite debería aumentar la fuerza extra hecha por la columna de aceite. Esta IF pone a disposición de C un elemento para la Observación de la ES; Observación que, como veremos más adelante, no les permite modificarla.

Los estudiantes prosiguen su indagación, apelando a las ecuaciones. Calculan las diferentes contribuciones al empuje, para sumarlas con el peso de la pelota, y además una fuerza extra, que es el peso de la columna de aceite. Es decir: la ES vuelve a ser la que era en un comienzo.

T12[ENT] ¿Qué han escrito hasta ahí?

T13 [C] [señalando el papel] La fuerza del peso, el empuje que hace el agua, y el empuje que hace el aceite.

T14[ENT] Y ¿por qué has escrito esas tres fuerzas? ¿quién hace cada una de esas tres fuerzas [IF]

T15[C] La Tierra [ENT repite en voz baja: la Tierra...], el agua, y

T16[ENT] [interrumpe] ¿Por qué el agua hace una fuerza sobre la pelota? [IF]

T17[C] Porque está sumergida... en el agua.

T18[ENT] [el agua] ¿está en contacto con la pelota? [C asiente], ¿por la presión? [C y N asienten] [...] ¿y la otra fuerza? [IF]

$\mathbf{T 1 9 [ C ]} Y$, el aceite...

T20[ENT] Y ¿por qué el aceite puede hacer una fuerza sobre la pelota? [IF]

T21 [C] [mirando a Ent] porque está sumergida la pelota en el aceite.

T22[ENT] Y ahora, ¿qué otra fuerza querés poner?

T23[C] Quiero poner, la columna de aceite, que está sobre mi pelota...

T24[ENT] ¿Y por qué la columna de aceite hace una fuerza sobre la pelota? [IF]

T25[C] Porque está encima de la pelota...

T26[ENT] Porque pega sobre las paredes de la pelota? ... hace presión? [Ambos asienten][...] Pero esa fuerza no la habían tenido en cuenta acá ya? [señala los términos de la fuerza de empuje] [IF] T27[C] iNo, no! no, no se [con duda, y girando la cabeza hacia N]

$\mathbf{T 2 8}[\mathbf{N}]$ Es que lo que no sé es si ese es el valor [toma el lápiz y señala el término de la fuerza 'extra', ambos quedan en silencio]

Lo primero en notar aquí es que el discurso ya no es progresivo, como lo fue hasta el turno 11. La ES volvió a ser la original: sobre la pelota hay tres fuerzas, independientes: su Peso, Empuje y Peso de la columna de aceite. Los estudiantes ya no Observan estas ideas, no incorporan nuevas Inferencias, ni desplazan las que le dan forma a la ES.

Al mismo tiempo, vemos que las IF siguen estando, pero ya no cumplen el rol de abrir camino para las observaciones de los estudiantes, ni de aportar elementos potencialmente útiles para ser incorporados como Inferencias a la ES. En términos discursivos no aportan a la progresividad. No facilitan que los estudiantes observen la ES, ni que incorporen alguna inferencia.

Pocos minutos después, los estudiantes deciden abandonar la resolución.

\section{Conclusiones}

El primer objetivo planteado en este trabajo fue el de caracterizar los tipos de intervenciones utilizadas durante una entrevista clínica. En ese sentido, se describen 
cuatro tipos de intervenciones, de Empatía (IE), de Socialización (IS), de Introducción (II) y de Focalización (IF). Las dos últimas, (II, IF), con potencialidades particulares para la necesaria improvisación que el entrevistador debe llevar a cabo, de modo de poder conducir la indagación hacia aquél terreno en el cual es más factible obtener datos útiles para la investigación.

En segundo lugar, se pudo llevar a cabo un análisis comparativo, entre dos entrevistas disonantes en cuanto a la calidad de los datos obtenidos, y caracterizar así la manera en que esas intervenciones se articulan con el discurso de los estudiantes. Mediante este análisis se describen formas en las cuales las II e IF permiten (o no) al entrevistador proveer elementos que los estudiantes pueden incorporar como Inferencias a la Estructura Semántica (ES), o abrir caminos de Observación. La Entrevista 1 constituye un caso exitoso en términos de la promoción de la progresividad discursiva, mientras que la 2, no. En ambas entrevistas, los mismos tipos de intervenciones, aún con el mismo rasgo distintivo de introducir elementos nuevos en el discurso (II) o de resaltar aspectos soslayados (IF), pueden tener efectos claramente diferentes en tanto y en cuanto promuevan (o no) la Observación de la ES que los entrevistados negocian durante la entrevista.

En relación a este análisis comparativo (entre ambas entrevistas) cabe resaltar el valor metodológico aportado por el marco interpretativo de la progresividad discursiva. Teniendo en cuenta solamente la caracterización de las intervenciones, no resulta posible entender la diferencia entre, por ejemplo, preguntar (Entrevista 1, turno 20) si hay empuje sobre la esfera, y no sobre el cubo, y preguntar (Entrevista 2, turno 26) si la columna de aceite hace fuerza sobre la pelota por estar en contacto ella. En ambos casos, la intervención focaliza en algo que ya está incorporado en la discusión (en un caso la diferente geometría de los cuerpos y en el otro el hecho de que el empuje actúa sobre el cuerpo vía el contacto) Estas intervenciones no se diferencian en la forma (ambas son IF), sino en el vínculo con la ES que los estudiantes están negociando. En el primer caso la intervención se conecta directamente con la Observación de que el empuje no puede depender de la geometría. En el segundo caso, la IF no se conecta con (y debería) la posibilidad de Observación de una característica fundamental de la ES: el peso de la columna de aceite es independiente de las demás fuerzas.

La habilidad para conducir entrevistas clínicas, con la ineludible necesidad de improvisar preguntas durante las mismas, se construye a través de la práctica. Tener en cuenta la manera en que esas intervenciones pueden articularse (o no) con la progresividad del discurso de los entrevistados, puede tener efectos disímiles en los datos obtenidos. Intervenir de manera sintonizada con la progresividad discursiva de los estudiantes parece ser una decisión metodológicamente potente para obtener datos útiles para el estudio del aprendizaje.

Las diferentes maneras en las que las intervenciones del entrevistador articulan con el discurso de los estudiantes no sólo permiten entender mejor el devenir del mismo durante una entrevista. También es importante preguntarse si existen similitudes entre estas articulaciones discursivas y las que tienen lugar en las aulas, no ya con las intervenciones de un entrevistador, sino de un docente. En principio es posible reconocer que durante la entrevista, y en el marco de ese escenario discursivo, los estudiantes se enfrentan con obstáculos para comprender y describir situaciones y 
utilizar conceptos. Como hemos visto, si bien el entrevistador no tiene en agenda un objetivo de enseñanza, gestiona discursivamente de maneras que pueden favorecer o no que los entrevistados superen esos obstáculos y avancen en su comprensión. Esta gestión discursiva tiene puntos en común con algunas de las funciones que los docentes llevan adelante en las aulas. En ese sentido, y aún cuando escapa a los objetivos planteados en este estudio, los resultados obtenidos en relación a las situaciones de entrevista podrían, en el futuro, ser un aporte para analizar las interacciones en las aulas.

\section{Referencias}

BEREITER, C. Implications of postmodernism for science, or, science as progressive discourse. Educational Psychologist, Philadelphia, v. 29, n. 1, p. 3-12, 1994. DOI: https://doi.org/10.1207/ s15326985ep2901_1.

BROWN, N.; DANISH, J.; LEVIN, M.; DiSESSA, A. Competence reconceived: the shared enterprise of knowledge analysis and interaction analysis. In: DiSESSA, A.; LEVIN, M.; BROWN, N. (ed.). Knowledge and interaction. New York: Routledge, 2016. p.11-29.

BROWN, S.; MONTFORT, D.; PEROVA-MELLO, N.; LUTZ, B.; BERGER, A.; STREVELER, R. Framework theory of conceptual change to interpret undergraduate engineering students' explanations about mechanics of materials concepts. Journal of Engineering Education, Hoboken, v. 107, n. 1, p. 113-139, 2018. DOI: https://doi.org/10.1002/jee.20186.

BUTELER, L.; COLEONI, E. Sobre el aprendizaje (y la enseñanza) del concepto de empuje: un análisis de la dimensión cognitiva y comunicativa de lo que ocurre durante una entrevista grupal. Revista de Enseñanza de la Física, Córdoba, Argentina, v. 26, n. extra, p. 17-28, 2014. Recuperado el 25 Feb. 2021 de: https://cutt.ly/AzReiKc.

CARRAHER, D.; SCHLIEMANN, A. The transfer dilemma. Journal of the Learning Sciences, Philadelphia, v. 11, n. 1, p. 1-24, 2002.

CHI, M.T.H. Quantifying qualitative analyses of verbal data: a practical guide. Journal of the Learning Sciences, Philadelphia, v. 6, n. 3, p. 271-315, 1997.

CLARÀ, M. Building on each other's ideas: a social mechanism of progressiveness in whole-class collective inquiry. Journal of the Learning Sciences, Philadelphia, v. 28, n. 3, p. 302-336, 2019.

COLEONI, E.; BUTELER, L.; BAUDINO, N. B. La entrevista clínica como método de indagación: algunas consideraciones metodológicas. Revista de Enseñanza de la Física, Córdoba, Argentina, v. 28, n. extra, p. 163-169, 2016. Recuperado el 25 Feb. 2021 de: https://revistas.unc.edu.ar/index. $\mathrm{php/revistaEF/article/view/15635.}$

DiSESSA, A. An interactional analysis of clinical interviewing. Cognition and Instruction, Philadelphia, v. 25, n. 4, p. 523-565, 2007. DOI: https://doi.org/10.1080/07370000701632413.

DiSESSA, A.; SHERIN, B. What changes in conceptual change? International Journal of Science Education, Abingdon, v. 20, n. 10, p. 1155-1191, 1998. DOI: https://doi. org/10.1080/0950069980201002.

ELLIS, A. The influence of reasoning with emergent quantities on students' generalizations. Cognition and Instruction, Philadelphia, v. 25, n. 4, p. 439-478, 2007. Recuperado el 25 Feb. 2021 de: https://www.jstor.org/stable/27739866.

ERICSSON, K. A.; SIMON, H. A. Protocol analysis: verbal reports as data. Cambridge, Mass.: MIT Press, 1993. 
GOFFMAN, E. Frame analysis. New York: Harper Colophon Books, 1974.

GOLDSTONE, R. L.; WILENSKY, U. Promoting transfer by grounding complex systems principles. Journal of the Learning Sciences, Philadelphia, v. 17, n. 4, p. 465-516, 2008. DOI: https://doi. org/10.1080/10508400802394898.

HALLDÉN, O.; HAGLUND, L.; STROMDAHL, H. Conceptions and contexts: on the interpretation of interview and observational data. Educational Psychologist, Philadelphia, v. 42, n. 1, p. 25-40, 2007. DOI: https://doi.org/10.1080/00461520709336916.

HAMILTON, D. J. The peer interview about complex events: a new method for the investigation of preinstructional knowledge. International Journal of Science Education, Abingdon, v. 18, n. 4, p. 493-506, 1996. DOI: https://doi.org/10.1080/0950069960180407.

IZSÁK, A. "You have to count the squares": applying knowledge in pieces to learning rectangular area. The Journal of the Learning Sciences, Philadelphia, v. 14, n. 3, p. 361-403, 2005. Recuperado el 25 Feb. 2021 de: https://www.jstor.org/stable/25473489.

LAMBERG, T. D.; MIDDLETON, J. A. Design research perspectives on transitioning from individual microgenetic interviews to a whole-class teaching experiment. Educational Researcher, Thousand Oaks, CA, v. 38, n. 4, p. 233-245, 2009. DOI: https://doi.org/10.3102/0013189X09334206.

LEMMER, M.; KRIEK, J.; ERASMUS, B. Analysis of students' conceptions of basic magnetism from a complex systems perspective. Research in Science Education, Dordrecht, v 50, p. 375-392, 2018. DOI: https://doi.org/10.1007/s11165-018-9693-z.

MORTIMER, E.; SCOTT, P. Meaning making in secondary science classrooms. Maidenhead: Open University Press, 2003.

MORTON, T. Classroom talk, conceptual change and teacher reflection in bilingual science teaching. Teaching and Teacher Education, Oxford, v. 28, n. 1, p. 101-110, 2012. DOI: https://doi. org/10.1016/j.tate.2011.07.006.

NAVARRO, M. Evolutionary maps: a new model for the analysis of conceptual development, with application to the diurnal cycle. International Journal of Science Education, Abingdon, v. 36, n. 8, p. 1231-1261, 2014. DOI: https://doi.org/10.1080/09500693.2013.855343.

ODDEN, T. B.; RUSS, R. S. Vexing questions that sustain sensemaking. International Journal of Science Education, Abingdon, v. 41, n. 8, p. 1052-1070, 2019. DOI: https://doi.org/10.1080/095006 93.2019.1589655.

PAPAGEORGIOU, G.; JOHNSON, P. Do particle ideas help or hinder pupils' understanding of phenomena? International Journal of Science Education, Abingdon, v. 27, n. 11, p. 1299-1317, 2005. DOI: https://doi.org/10.1080/09500690500102698.

PIAGET, J. The child's conception of the world. London: Kegan Paul, Trench \& Trubner, 1929.

PIAGET, J. The language and thought of the child. London: Kegan Paul, Trench \& Trubner, 1926.

POSNER, G. J.; GERTZOG, W. A. The clinical interview and the measurement of conceptual change. Science Education, Hoboken, v. 66, n. 2, p 195-209, 1982. DOI: https://doi.org/10.1002/ sce.3730660206.

ROTH. W.-M. The nature of scientific conceptions: a discursive psychological perspective. Educational Research Review, Amsterdam, v. 3, n. 1, p. 30-50, 2008. DOI: https://doi.org/10.1016/j. edurev.2007.10.002.

RUSS, R. S.; LEE, V. R.; SHERIN, B. L. Framing in cognitive clinical interviews about intuitive science knowledge: dynamic student understandings of the discourse interaction. Science Education, Hoboken, v. 96, n. 4, p. 573-599, 2012. DOI: https://doi.org/10.1002/sce.21014. 
RUSS, R.; SHERIN, B.; LEE, V. The intersection of knowledge and interaction: challenges of clinical interviewing. In: DiSESSA, A.; LEVIN, M.; BROWN, N. (ed.). Knowledge and interaction. New York: Routledge, 2016. p. 377-402.

SHABAN, Y.; WILKERSON, M. The co-construction of epistemological framing in clinical interviews and implications for research in science education. International Journal of Science Education, Abingdon, v. 41, n. 12, p. 1579-1599, 2019. DOI: https://doi.org/10.1080/09500693.2019.1620972.

TABER, K.; GARCIA-FRANCO, A. Learning process in chemistry: drawing upon cognitive resources to learn about the particulate structure of matter. Journal of the Learning Sciences, Philadelphia, v. 19, n. 1, p. 99-142, 2010. DOI: https://doi.org/10.1080/10508400903452868.

VOLFSON, A.; ESHACH, H.; BEN-ABU, Y. When technology meets acoustics: students' ideas about the underlying principles explaining simple acoustic devices. Research in Science Education, Dordrecht, 2020. DOI: https://doi.org/10.1007/s11165-019-09913-w. 